Insgesamt motivierte die Leitfrage „Wann ist genug genug?" zu einer intensiven Reflexion. Die Diskussion zeigte, dass der Adressat für Fragen und Wissenslücken nicht länger ausschließlich die jetzige Art der Forschung und Wissenschaft sein kann. Andererseits sollten Hersteller auch keine Lösung durch konkrete Richtlinien aus der Politik erwarten, sondern durch verstärkten Austausch mit allen Akteuren einen Abgleich der dringendsten Punkte gemeinsam erreichen. Prinzipiell liegt zwar ein beachtliches Wissen über Verhalten und Wirkung von Nanomaterialien vor, dieses wird jedoch nicht effizient und verständlich genug zwischen Wissenschaft und Regulatoren ausgetauscht und für eine Korrektur der Forschungsagenda genutzt. Der Workshop bot eine gute Gelegenheit, diesen Austausch anzustoßen und zu üben, die Probleme der jeweils anderen in der eigenen Agenda zu berücksichtigen. Damit wurde deutlich, dass in der Weiterentwicklung der festgefahrenen Beziehung zwischen Wissenschaft und Politik ein wichtiger Lösungsansatz zu finden ist: nicht für alle Probleme, aber genug für den Anfang!

Die Statements wurden in einer abschließenden Forumsdiskussion zusammengeführt und sollen in einem Synthesedokument in Kürze publiziert werden.

$\ll »$

\section{Grenzenloses Experimentieren?}

Bericht zum Workshop „Experimentelle
Gesellschaft - das Experiment als wis-
sensgesellschaftliches Dispositiv?“

Karlsruhe, 20.-21. Februar 2014

von Stefan Böschen, ITAS, Matthias Groß, UFZ Leipzig/Friedich-Schiller-Universität Jena, und Wolfgang Krohn, Universität Bielefeld

Experimentieren ist in. Reallabore sollen eingerichtet werden, Klimaexperimente scheinen allgegenwärtig und politische Experimente sowieso. Auf dem Workshop „Experimentelle Gesellschaft: Das Experiment als wissensgesellschaftliches Dispositiv?" wurden Spielarten und Erklärungsmöglichkeiten des Experiment-Be- griffs ausgelotet. In seinem Einleitungsreferat hob Wolfgang Krohn hervor, dass Experiment und Modernität spätestens seit der Renaissance eng miteinander verschränkt sind, sich aber die Formen dieser Verbindung zu verändern scheinen. Wurde einst die Grenze zwischen Wissenschaft und Gesellschaft hochgehalten, so vollzogen sich im Laufe der Modernisierung immer neue Freisetzungsversuche des Experimentalismus bis dahin, dass in der Gegenwart experimentellem Forschen ein Tätigkeitsmodus außerhalb der Wissenschaft zuerkannt wird. Um diesen Rahmen zwischen Experimenten außerhalb und innerhalb von Gesellschaft auszuleuchten, unterteilte sich der Workshop in vier thematische Diskussionseinheiten, die im Folgenden nachgezeichnet werden.

\section{Das Experiment als Kollektivsymbol gesellschaftlicher Modernität}

Im ersten Themenblock stand das Interesse, den semantischen Raum des Experimentierens im Spiegel öffentlich-medialer Kommunikation auszuleuchten, um dabei zu Kennzeichnungen von politischen Revolutionen, kulturellen Epochenbrüchen, polit-ökonomischen oder technologischen Veränderungen zu gelangen. Rolf Parr (Universität Duisburg-Essen) verwies in seinem Beitrag „Experiment. Kollektivsymbol, Narrativ und Modernitätsindikator" zunächst auf drei Bedeutungsgehalte, in denen von Experimenten gesprochen wird, eine engere, auf das wissenschaftliche Labor bezogene Forschungstätigkeit, eine Erfassung aller weiteren Formen des Experimentierens sowie eine metaphorische Verwendung zur Kennzeichnung riskanter Unternehmungen. Davon ausgehend arbeitete Parr anhand zweier Reden von Merkel zu Europa heraus, wie je nach Kontext der Verweis auf „Experiment" eine positive oder negative Konnotation erhalten kann und erläuterte vor dem Hintergrund der Interdiskurstheorie, wie das Experiment als Thematisierungsform kollektivsymbolisch wirkt und unterlegte dies mit Ausdeutungen zum „Experiment Griechenland“. Nina Janich (TU Darmstadt) untersuchte in ihrem Beitrag „Zur öffentlichen Semantik des Experiments" anhand des Digitalen Wörterbuchs der Deutschen Sprache (DWDS) 
das semantische Feld des Begriffs Experiment. Überraschend waren die sich abzeichnenden historischen Konjunkturen in der Häufigkeit der Wortverwendung, aber auch die jeweilige Geschichte von Assoziationen mit bestimmten weiteren Wörtern. Am Beispiel des LOHAFEX-Experiments zur Meeresdüngung des Südatlantiks kontrastierte sie schließlich, wie Experiment als Schlüsselwort und Kristallisationspunkt öffentlicher Kontroversen um Wissenschaft fungiert, indem es als Instrument zur Genese von Wissen dargestellt, oder eben zur Einforderung von Legitimation von Forschung thematisiert wird. Diese Diskussionseinheit machte deutlich, dass die Entwicklung semantischer Konnotationen als eine Geschichte von Konjunkturen in den Verwendungskontexten erzählt werden kann.

\section{Laboratorien der Gesellschaft}

Laboratorien des Suchens lassen sich viele finden. Aber: Welche Ausdeutungen, Selbst- und Fremdzuschreibungen des Experimentellen lassen sich dabei ausmachen? In zwei Keynotes und einem Expertenpanel wurde diese Frage facettenreich behandelt. Den Start in die Diskussionseinheit vollzog Holger Schulze (Sound Studies Lab Berlin) mit seinem Beitrag „Im Schmutzraum. Über explorative Praktiken der Gegenwartskunst“. Er positionierte seiner Überlegungen über die prekären Projektionen zwischen den beiden Sphären Wissenschaft und Kunst. Danach wird die Wissenschaft mit Klarheit und Reinheit assoziiert, die Kunst erscheint eher als ein „Schmutzraum“. Schulze, der den Begriff des Experiments mit dem der Heuristik verband, erläuterte schließlich an unterschiedlichen Exponenten der Gegenwartskunst sein Verständnis von künstlerischen Experimenten als zeitgebundene künstlerische Generativität. Marcus Andreas (RCC München) lenkte mit seinem Bericht zu ,Soziotope des Experimentierens. Vom Ökodorf zum Environmental Citizenship?" den ethnologischen Blick auf das Ökodorf Siebenlinden als Experimentierraum, in dem verschiedene Stadien des Experimentierens mit einer neuen Lebensform auftraten. Der experimentelle Charakter erschloss sich im Blick auf das ganze Setting von Handlungsentwürfen und ihrer Überprüfung, die bei der Konturierung dieser modellhaften Lebensform erprobt wurden. Neben diesen beiden Keynotes wurden im Rahmen eines Expertenpanels unterschiedliche Formen und Orte gesellschaftlichen Experimentierens in den Blick genommen. Lisa-Marian Schmidt (Alice Salomon Hochschule Berlin) zeigte in ihrem Beitrag „Experimentalsettings zur Selbsterkundung und Kompetenzfeststellung im Bildungssystem" auf, wie unter dem Eindruck von Pisa neue Formen der Bildungsmessung Einzug halten und das Kompetenzdispositiv wirksam ist. Jürgen Howaldt, Ralf Kopp und Michael Schwarz (TU Dortmund) führten mit ihrem Beitrag „Experimentelle soziale Praktiken als Treiber des sozialen Wandels. Überlegungen zu einer Theorie sozialer Innovation" aus, dass das wachsende Interesse an kollektiven Experimenten auf die Herausbildung eines neuen Innovationsparadigmas verweise. Sie argumentierten für eine Theorie sozialer Innovation, in deren Kern experimentelle soziale Praktiken stehen. Stephan Lingner (Europäische Akademie Bad Neuenahr-Ahrweiler) verwies in seinen Überlegungen zu „Climate Engineering als gesellschaftliches Experiment" darauf, dass im Kontext des Klimawandels der Experimentbegriff in unterschiedlichen Schattierungen verwendet werde und deshalb zwischen globalen/ lokalen Settings einerseits und unbeabsichtigten/ beabsichtigten Aktivitäten unterschieden werden müsse. Das Algen-Düngungsexperiment sei danach ein Experiment, da es beabsichtigt stattfand und den globalen Problemhorizont adressiere. In seinem Beitrag „Spielerisch zum Wandel - Reallabor ,Bürgerbörse“" ging Stefan Selke (Hochschule Furtwangen) in die Details einer kollektiven Antragstellung, die sich vor dem Hintergrund der Herausbildung eines Reallabors „Bürger-Börse“ in Furtwangen abspielte. Im Mittelpunkt stand dabei, dass die Forschungsthemen möglicher Realexperimente in einer Bürgerbörse nicht nur präsentiert, sondern dort auch durch die Zuweisung von „Bregtalern“ (jeder Bürger erhielt 100) bewertet und entsprechend mit Geld ausgestattet wurden. Diese Diskussionseinheit führte eine Fülle an Formen experimenteller Praktiken vor, verwies jedoch auch auf die Aufgabe, konzeptionell die Konturen des Experimentbegriffs zu schärfen, um sich nicht in der Vielfalt an Verwendungsformen zu verirren. 


\section{Experimentelle Governance}

Dieser Themenblock gruppierte sich um experimentelle Praktiken, die mit Eingriffen in Rechte Betroffener, anerkannte Interessen, basale Werte oder eben in die Natur verbunden sind. Entsprechend ist das Feld des Experimentellen auch ein Feld des Politischen. Thomas Saretzki (Leuphana Universität Lüneburg) verwies in seiner Keynote darauf, dass es schon in den ausgehenden 1970er und beginnenden 1980er Jahren eine Debatte über Experimentelle Politik in der Politikwissenschaft gab. Rückblickend lässt sich fragen, was daran Rhetorik im Zuge der Reformbewegungen war, oder darüber hinaus neue Verfahren zur systematischen Erfahrungs- und Erkenntnisgewinnung waren. Vor diesem Hintergrund sollte zwischen Modellpolitik, die Reformziele und -strategien betreibt, und Experimenteller Politik, in der es zusätzlich um Lernfähigkeit und Lernbereitschaft geht, unterschieden werden. Peter Wehling (Johann Wolfgang Goethe-Universität Frankfurt a. M.) führte in seinen Überlegungen zu „Experimentelle Governance des Nichtwissens" einen analytischen Experimentbegriff ein, unter den ein solches Handeln fällt, das die Bedingung einer lernbereiten Beobachtung von Handlungsfolgen erfüllt. Darin sind epistemische (Beobachtbarkeit, Zurechenbarkeit) und normative Implikate (Lernbereitschaft, Verantwortbarkeit) miteinander verbunden. Eine experimentelle Governance des Nichtwissens muss sich auch daran bemessen lassen, klare Regeln für den Abbruch von Experimenten (z. B. unter Gefährdungsgesichtspunkten) als legitime Option zu benennen. Auf dem Expertenpanel verwiesen Andreas Lösch und Christoph Schneider (ITAS/KIT) mit ihren Überlegungen zu „Die interdependenten Experimente der Energiewende" darauf hin, dass die Energiewende ein besonderes Modernisierungsprojekt darstelle, da hier ein Netz ganz heterogener Elemente durch die Experimentalisierung ihrer Verknüpfungen neu geordnet würde. Zukunftsvisionen eröffnen dabei die Chance, alte, neue und noch gar nicht etablierte Elemente zu verknüpfen. Fritz Reusswig (PIK Potsdam) führte in die „Urbs Laborans Berlin" ein. Hintergrund ist ein Gutachten für die Stadt Berlin zu Möglichkeiten einer Dekarboni- sierungsstrategie. Deutlich wurde, an wie vielen Ansatzpunkten eine Stadt gleichzeitig kleinteilige Experimente vollziehen muss, um ihr ehrgeiziges Ziel der Klimaneutralität erreichen zu können. Im gleichen Themenfeld, aber im transnationalen Raum positionierte Arno Simons (TU Berlin) seine Überlegungen zu „What can we learn from the grand policy experiment?"“. Dabei verwies er auf eine Experimentkette im Feld des Emissionshandels und fragte danach, was die Rolle von Wissenschaftlern als kritische Beobachter sei. Er beklagte, dass ein relativ strenger analytischer Experiment-Begriff, der von der Wissenschaft hoch gehalten werden sollte, durch einen lockeren alltagssprachlichen Gebrauch verwässert würde. In dieser Diskussionseinheit zeigte sich, dass gerade weil gegenwärtig viel von Transformationen die Rede sei, Praktiken der Lernbereitschaft wie ihrer politischen Institutionalisierung in ihrer Bedeutung und Reichweite genau abgewogen werden müssen. Auch wenn sie rhetorisch gerne bemüht werden, so sind die Differenzen zwischen Talk und Action gerade in diesem Feld oft immens. Deshalb drängt sich die Fragen auf, einerseits was die Berufung auf das Experimentelle politisch bedeutet und andererseits wie in im Regelkanon des demokratischen Rechtsstaats gestaltet werden kann.

\section{Philosophie des Experimentalismus}

Welche Bedeutung kommt neuen Wissenstechnologien bei der Konstitution des Experimentellen zu? Auf dem Expertenpanel führte Petra Ahrweiler (Europäische Akademie Bad Neuenahr-Ahrweiler) zunächst in die Methodologie von "Simulationsexperimenten zur Innovation in Netzwerken“ ein. Diese Methodologie erlange an Bedeutung durch die Tatsache, dass mittels Simulationen partizipative „Echtzeit-Entscheidungsfindungen" exploriert werden könnten. Stephan Lorenz (Friedrich-Schiller-Universität Jena) ging mit seinen grundsätzlichen Überlegungen zu „Experimente als Verfahren - eine prozedurale Perspektive" auf Fragen und Problemstellungen des Demokratischen Experimentalismus ein und argumentierte dafür, das Augenmerk auf Prozeduralität zu legen. Cornelius Schubert (Universität Siegen) vertrat in seinem Beitrag „Experimen- 
telle Zukünfte. Numerische Prognosen als Instrumente generativen Zukunftswissens" die These, dass das Experimentaldispositiv sich über zirkulierende Idee-Instrument-Pakete konstituiere und stabilisiere. Tanja Bogusz (HU Berlin) führte schließlich zu „Experimentalismus als Mittel sozialwissenschaftlichen Erklärens" aus. Sie argumentierte für eine Soziologisierung von Deweys Forschungstheorie, so dass Theorie nicht als feste sondern als explorative Größe gehandelt wird.

\section{Fazit}

Was kann zusammenfassend für die weitere Diskussion besonders empfohlen werden?

Erstens lässt sich aufweisen, dass Experimentieren einen wesentlichen Produktionsmodus von Wissen darstellt und mit der Etablierung dieser Handlungspraxis wurden institutionelle Grenzen der Wissenschaft gezogen, die sich immer weiter in die Gesellschaft hinein schieben. Jedoch lässt sich nicht behaupten, dass es gleichsam eine lineare Ausbreitungsgeschichte zu erzählen gilt. Vielmehr zeigen Sprachanalysen, dass die Nutzung des Experiment-Begriffs Konjunkturen unterlag und unterliegt. Insofern ist es aufschlussreich, diese Konjunkturen nachzuzeichnen, um die Verbreitung des ExperimentBegriffs genauer kontextualisieren zu können.

Zweitens hat sich auf dem Workshop gezeigt, dass die weite Verwendung des Experiment-Begriffs zunächst eine Debatte eröffnet, weil er dazu einlädt, verschiedene Praktiken des Ausprobierens in den Blick zu nehmen. Jedoch stellt sich in einem nächsten Schritt die Herausforderung, den analytischen Gebrauch des Experiment-Begriffs von einem empirischen zu unterscheiden und ihn kriteriell abzusichern.

Drittens wurde deutlich, dass mit dem Experiment-Begriff gezielt Settings gesellschaftlichen Erprobens untersucht werden können, bei denen es zu einer Kooperation von heterogenen Akteuren kommt. „Soziale Innovationen“ oder „Reallabore" sind hierauf bezogene konzeptionelle Annäherungen, Energiewende, Climate Engineering und Ökodörfer einschlägige Phänomene.

Viertens zeigte sich, dass mit dem Experiment-Begriff die neuen Beobachtungsverhältnisse thematisiert werden. Denn wer sind die
Akteure des Experimentierens? Man könnte es so formulieren: Die Innovationsdynamik der Gesellschaft war schon immer von Realexperimenten durchzogen, doch in der Gegenwart werden die methodologischen Bedingungen sowie die Begründungs- und Rechtfertigungsverhältnisse neu austariert.

Die Tagung hat verdeutlicht, dass es einer Fülle von interessanten Fragen nachzugehen gilt. Diese haben zugleich für die Technikfolgenabschätzung einigen Belang. Denn mit dem Übergang von einer Politik- zur Gesellschaftsberatung lässt sich auch argumentieren, dass dies einen Übergang zu Verfahren experimenteller Erprobung bedeutet, für die politisch die entsprechenden institutionellen Rahmenbedingungen geschaffen werden müssen. Der Technikfolgenabschätzung fiele dann die Aufgabe zu, nicht allein „Blaupausen“ für die soziale Organisation solcher Prozesse zu entwickeln, sondern insbesondere auch den Aspekt des Wissenserwerbs systematisch zu unterstützen.

Der Workshop hat erbracht, dass die Betrachtung des Experiments als wissensgesellschaftliches Dispositiv analytisch aufschlussreich ist. Es wurde zur Vernetzung dieses Diskussionszusammenhangs eine moderierte Mailingliste mit Archiv angelegt, um die Aktivitäten nicht nur zu dokumentieren, sondern zugleich gezielt weitertreiben zu können. Bei Interesse bitte melden bei: stefan.boeschen@kit.edu.

$\langle\gg$

\section{Hummeln und Schmetterlinge im Open Space}

\section{Bericht von der Werkstatttagung der \\ Evangelischen Akademie Loccum zu Partizipation \\ Loccum, 28.-30. Januar 2014 \\ von Elske Bechthold und Stefanie B. Seitz, ITAS}

So vage die Bedeutung des Begriffs auch sein mag, Bürgerbeteiligung liegt im Trend. Die TAForschung beschäftigt sich schon seit mehreren Jahrzenten mit dem Thema Partizipation - am 